\title{
Applications of Spherical Aberration Correction in STEM and TEM
}

\author{
S. Lazar***, J, Etheridge***, C. Dwyer***, B. Freitag**, and G. A. Botton****** \\ * Canadian Centre for Electron Microscopy, McMaster University, Hamilton Ontario, \\ Canada \\ ** FEI Electron Optics, 5600 KA Eindhoven, The Netherlands \\ *** Monash Centre for Electron Microscopy and Department of Materials Engineering, \\ Monash University, Victoria 3800, Australia \\ **** Department of Materials Science and Engineering, McMaster University, Hamilton, \\ Ontario, Canada
}

Recent developments in ultra stable electronics and computer controlled systems have made possible a significant step forward in electron microscopy through the introduction of aberration corrected microscopes [1-2]. With an achievable resolution up to $0.5 \AA$ in TEM and STEM [3], aberration corrected microscopes have introduced the possibility of measuring atom position and atom type with unprecedented resolution [4,5]. The improved resolution, especially in the STEM, brings new challenges in terms of understanding image formation and where within the specimen analytical signals are generated. Here we report some exploratory examples of atomic resolution EELS mapping that point out the difficulty of interpreting the results for some materials. In addition, we employ a double corrected microscope to image the probe and the real space distribution of the electrons in the specimen to better understand the spatial origin of the elastic and inelastic signals in images and chemical maps.

The experiments were carried out on a double corrected Titan 80-300 Cubed equipped with a monochromator. This instrument achieved an information limit of $0.65 \AA$, a STEM information transfer of better than $0.7 \AA$ and an energy resolution in EELS of $0.13 \mathrm{eV}$.

Elemental maps were acquired from a $\mathrm{LaB}_{6}$ specimen in $<100>$ orientation using two different collection angles with probe size smaller than $1.5 \AA$. When the experiment was carried out with a large collection angle $(110 \mathrm{mrad})$ a strong contrast could be obtained in the La N 4,5 map (fig 1b) but no contrast in the B K edge map (fig 1c). The lack of contrast in the B map could be explained by the fact that the ratio between elastic and inelastic scattering for La is high (a factor of about 3). Therefore, when the beam is on top of the La column, most of the electrons are elastically scattered on the neighboring B columns that generate a strong B signal comparable in intensity to the one generated when the beam is on the B column.

The situation is even further complicated when smaller collection angles are used for imaging. The spectrum-image data obtained with a $27 \mathrm{mrad}$ collection angle (something that would already be considered to be large) shows the volcano structure for the La $\mathrm{N}$ edge (fig 1e), while the La M edge signal extracted form the same spectra does not show such effect. The effect is in agreement with the theoretical predictions for this collection angle.

Using a double corrected microscope we can also image the electron probe not only in vacuum but also in the specimen without being affected by the aberrations of the imaging system. By scanning the probe across the specimen and collecting the real space intensity distribution of the electrons with a ring detector (fig.2a) we obtain atomic resolved images (fig. 2b). The probe was scanned across a specimen in steps of $0.2-0.5 \AA$. The scattered intensity of the probe in real space was recorded with a CCD camera, providing the 
scattered intensity distribution which reflect the local site symmetry of $\mathrm{Au}$ (fig.2d). Theoretical calculations predict that sub-Ångström electron probes will rapidly disperse onto atomic columns adjacent to the initial probe position [6]. The impact on this particular imaging configuration on the EELS signal, imaging and diffraction will be discussed.

[1] Haider, M., Rose, H., Uhlemann, S., Schwan, E., Kabius, B. and Urban, K. Ultramicroscopy, 75, (1998) 53-60.

[2] Krivanek, O. L., Dellby, N. and Lupini, A. R., Ultramicroscopy, 78, (1999) 1-11.

[3] C. Kisielowski, et al., Microscopy and Microanalysis 14 (2008) 454-462

[4] D. A. Muller, Nature Materials 8, (2009) 263-270.

[5] L. Gunawan, S. Lazar, O. Gautreau, C. Harnagea, A. Pignolet and G. A. Botton Applied Physics Letters, 95, (2009), 192902

[6] C Dwyer, J Etheridge, Ultramicroscopy 96 (2003), 343-360.
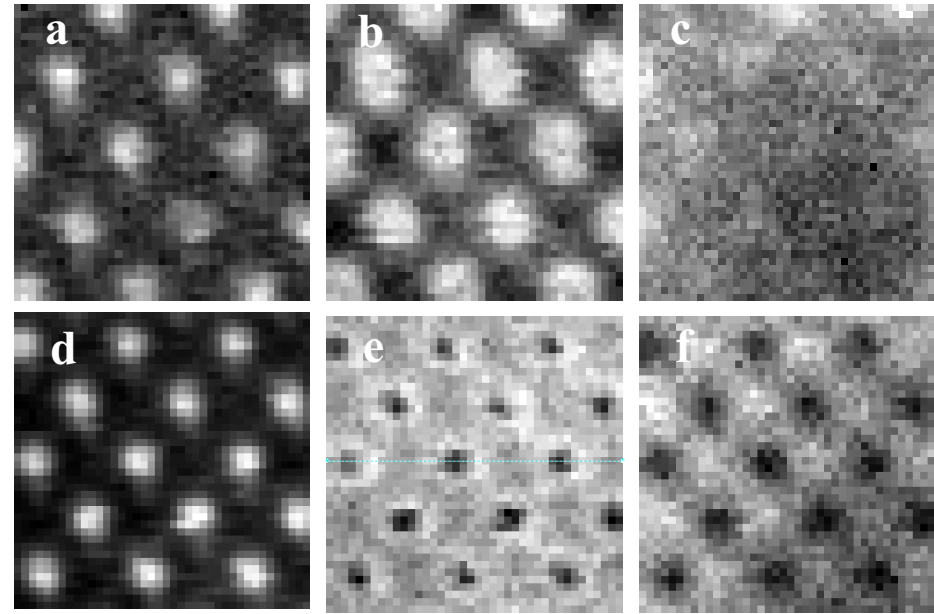

Figure 1. Atomic resolution EELS maps from $\mathrm{LaB}_{6}$ in [100] orientation. The experiment was performed at $200 \mathrm{kV}$ acceleration voltage, $17.6 \mathrm{mrad}$ convergence angle and $110 \mathrm{mrad}$ collection angle $(a, b, c)$ or $27 \mathrm{mrad}(\mathrm{d}, \mathrm{e}, \mathrm{f})$. a) and d) annular dark field image of the mapped area recorded simultaneous with the EELS signal, b) and e) $\mathrm{La} \mathrm{N}_{45}$-edge map, c)and f) B map, energy range $188-220 \mathrm{eV}$.

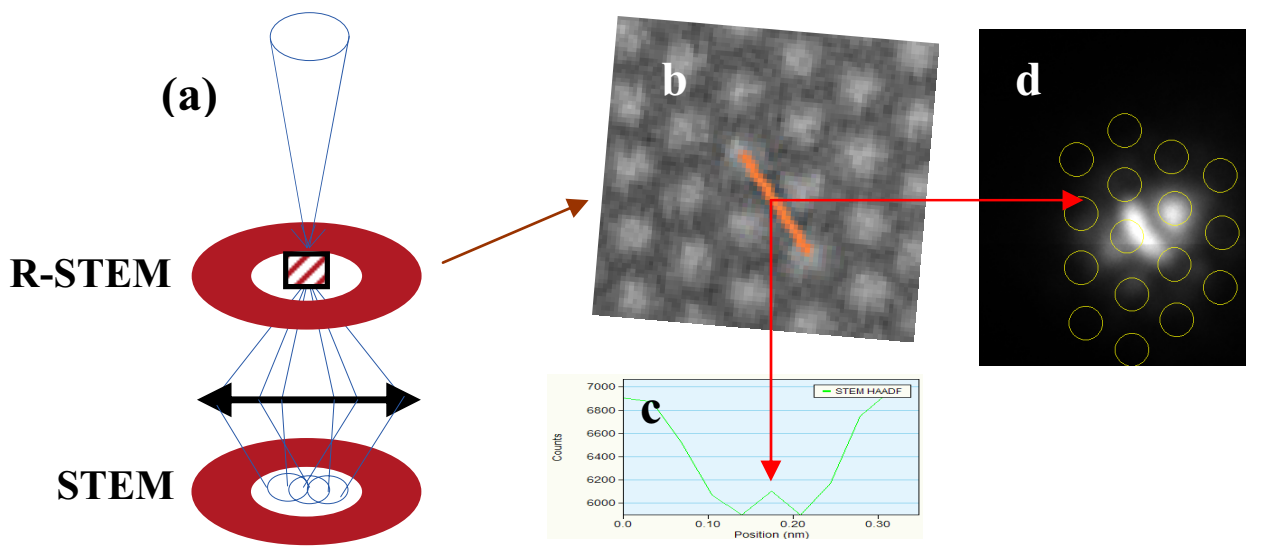

Figure 2. Image of scattered intensity distribution ( $\mathrm{d}$ ) from a specific site in $\mathrm{Au}<111>$ specimen. a) Real STEM (R-STEM) setup compared to conventional STEM. b) Image obtained in real space using the ring detector, c) Intensity profile along the line scan. d) Scattered intensity recorded on the CCD. The positions of the atomic columns are marked with yellow circles. 